\title{
Inclined Landing Testing of an Omni-Directional Unmanned Aerial Vehicle
}

\begin{tabular}{|c|c|}
\hline Journal: & Transactions of the Canadian Society for Mechanical Engineering \\
\hline Manuscript ID & TCSME-2017-0008.R1 \\
\hline Manuscript Type: & Article \\
\hline Date Submitted by the Author: & 28-Sep-2017 \\
\hline Complete List of Authors: & $\begin{array}{l}\text { von Frankenberg, Florentin; University of Ontario Institute of Technology, } \\
\text { Department of Automotive, Mechanical, and Manufacturing Engineering } \\
\text { Nokleby, Scott; University of Ontario Institute of Technology, Department } \\
\text { of Automotive, Mechanical, and Manufacturing Engineering }\end{array}$ \\
\hline $\begin{array}{l}\text { Is the invited manuscript for } \\
\text { consideration in a Special } \\
\text { Issue? : }\end{array}$ & CCTоMM 2017 Symposium Special Issue \\
\hline Keywords: & $\begin{array}{l}\text { Unmanned Aerial Vehicle (UAV), Quadrotor, Omni-Directional, Inclined } \\
\text { Landing }\end{array}$ \\
\hline
\end{tabular}

\section{SCHOLARONE \\ Manuscripts}




\title{
Inclined Landing Testing of an Omni-Directional Unmanned Aerial Vehicle
}

\author{
Florentin von Frankenberg; florentin.vonfrankenberg@uoit.ca \\ Mechatronic and Robotic Systems Laboratory, University of Ontario Institute of \\ Technology, Oshawa, ON, CANADA \\ Scott B. Nokleby; scott.nokleby@uoit.ca; Corresponding Author \\ Mechatronic and Robotic Systems Laboratory, University of Ontario Institute of \\ Technology, Oshawa, ON, CANADA
}

\begin{abstract}
Traditional multirotors and helicopters control translational movement by changing the orientation of the entire vehicle. This limits the effectiveness of such vehicles in applications as a mobile manipulator base. In these applications it is often necessary to fly in proximity to large structures where unpredictable aerodynamic conditions exist. In order to maintain precise control of position it is necessary to counteract disturbance forces quickly, and, due to the delay induced by rolling and pitching the entire vehicle, traditional multirotors and helicopters have a limited ability to maintain position precisely. Additionally, a mobile base must be capable of resisting arbitrary combinations of force and torque resulting from use of a manipulator arm. This is also not possible for traditional multirotors and helicopters. A novel UAV concept is presented which features the addition of four rotors directed orthogonally to the main lift rotors of a traditional quadrotor design. These rotors allow de-coupling of orientation from translational movement. Tests done on a physical prototype demonstrated the ability to sustain roll or pitch angles up to $15^{\circ}$ independently of translational movement, including the ability to move backwards at an angle and to dock on inclined surfaces in a controlled manner. The tests show that the OmniCopter can successfully land on surfaces with up to $30^{\circ}$ inclination. This type of motion is impossible for a traditional multirotor vehicle.
\end{abstract}

Keywords: Unmanned Aerial Vehicle (UAV); Quadrotor; Omni-Directional; Inclined Landing 


\section{Introduction}

This paper focuses on one component of a larger project in which an Aerial Manipulator System is to be developed. This project, described in detail in Baltovski et al. (2013) and Baltovski et al. (2015), requires an aerial platform capable of proximity flying and landing on structures. A diagram providing a conceptual overview of the larger project is shown in Fig. 1. The goal of the greater project is to develop a system capable of application in very remote areas for inspection, construction, and/or maintenance of infrastructure such as high-voltage power transmission lines, oil pipelines, or other similar structures. In the system proposed for this, a large lifting-gas 'mothership' will autonomously travel along the pipeline or power transmission line. The 'mothership' will supply power via a physical cable, denoted 'Umbilical Cable' in Fig. 1, to a smaller autonomous aerial vehicle which will conduct the actual inspection or servicing task. This paper focuses on the development of the smaller Unmanned Aerial Vehicle (UAV) at the end of the physical cable.

Several important concepts for aerial mobile manipulator systems are highlighted in Jiang (2013). First and foremost is the importance of force closure for manipulators. Force closure is defined as the ability of a mechanism to directly resist any arbitrary wrench, or combination of force and torque. This is an important property for dexterous manipulators. A mobile manipulator base must have force closure because forces exerted by a manipulator are transferred to the base. This is not a major issue for ground-based mobile manipulators due to contact with the ground providing frictional resistance to forces. Aerial manipulator bases cannot depend on friction with the environment to allow them to resist forces and torques.

Some important observations about traditional multirotors are also made. Since all rotors on traditional multirotors face in a parallel direction, they can only generate forces along this axis. If one designates this direction the $Z$-axis, then it can be said that generating linear forces along the $X$ and $Y$ directions is impossible for a traditional multirotor. Traditional multirotors can only pitch, roll, yaw, and lift. In order to move in the $X$ and $Y$ directions a traditional multirotor must first pitch or roll. This means that these systems are nonholonomic. The same holds true for helicopters. These characteristics make traditional multirotors and helicopters unsuitable for mobile manipulation.

Challenges arising from using nonholonomic bases for mobile manipulators are noted in Cano et al. (2013). In this paper a 6-DOF (degrees-of-freedom) manipulator for aerial mobile manipulation tasks was developed. The authors note that for rotary wing UAV, reaction torques caused by changes in the position of the manipulator's centre of gravity produce an inclination of the rotor's plane which also induces a displacement of the entire aerial platform. This is due to the coupled nature of orientation with translational motion for traditional multirotors and helicopters.

A number of novel UAVs have been developed to address some of the above limitations. In Albers 
et al. (2010), a single actuator was added to a traditional quadrotor design to allow their UAV to apply a horizontal force to vertical surfaces while in flight. This is used to facilitate tasks such as cleaning walls or windows. In order to overcome the limitations of traditional multirotors and helicopters, a novel hexrotor design was developed in Jiang (2013). In order to generate off-axis forces, the rotors' axes of thrust are canted by $30^{\circ}$ such that they are no longer parallel. This is very similar to Langkamp et al. (2011) except that in Langkamp et al. (2011) the rotors are canted at $45^{\circ}$ and variable pitch rotors are used as opposed to variable speed ones. In Langkamp et al. (2011), the authors indicate that the primary benefit is the ability to fly in arbitrary body attitudes. In Long and Cappelleri (2013), a UAV with five rotors was developed and constructed. A large central rotor is responsible for generating the majority of the lift while the three outer rotors are used for maneuvering. The three outer rotors can rotate into a configuration where their axes of thrust are perpendicular to the main lift rotor. In this work, simulations of circular path tracking were conducted and the authors note that the UAV is capable of tracking the circular path while maintaining zero attitude orientation. The authors note that quadrotors are limited due to being underactuated, that a sensor or gripper could not be arbitrarily oriented during flight, and that a quadrotor cannot hover in place with any body orientation.

In Salazar et al. (2009), an eight rotor UAV was developed with four rotors added outwards of a standard quadrotor design. The additional four rotors are facing perpendicular to the original four quadrotor rotors. The resulting system has translational and rotational dynamics almost completely decoupled, with the exception being that there is some aerodynamic interaction between the rotors due to the fact that the horizontally facing rotors direct an airstream directly underneath the lifting rotors.

In Brescianini and D'Andrea (2016), a fully omni-directional UAV with eight rotors is presented. The geometric configuration of rotors was chosen to maximize agility in any direction. Testing on a prototype vehicle demonstrated an ability to rotate about an axis independently of translational movement. The rotations were performed while maintaining a hover position with an error between 5 and $10 \mathrm{~cm}$. In another test, the UAV was commanded to track a circle of $1.5 \mathrm{~m}$ at $1.95 \mathrm{~m} / \mathrm{s}$, and was able to maintain a position accuracy between 10 and $15 \mathrm{~cm}$. In this test the results indicated a coupling between translational and rotational dynamics, potentially due to unmodelled aerodynamic interactions between rotors.

The ability to land on inclined surfaces with UAVs has been the topic of several research papers. Das et al. (2012) used four ultrasound sensors (one under each rotor) to develop a control strategy to land on oscillating surfaces. Based on the sensor readings the control strategy adjusts the roll, pitch, yaw, and throttle to compensate for the inclination of the landing surface. Papa and Core (2015) developed a similar system based on ultrasonic sensors to enable UAVs to land on inclined surfaces.

Dougherty et al. (2014) developed a strategy where a UAV flies over a proposed landing site. Lasers 
project bright points on the landing site and a CMOS camera is used to determine the coordinates of those laser points in the image plane. Based on these readings, the altitude of the laser modules can be determined and the orientation and distance of the landing site can be calculated. The system then performs an "aggressive landing trajectory" so that the UAV lands parallel to the surface.

Vlantis et al. (2015) used a camera system and a target on the landing surface to develop an algorithm to allow a UAV to land on a moving platform with an inclined surface.

Mizutani et al. (2015) designed a passive rotating spherical shell to go around a UAV. As the shell is a geodesic dome with facets and not a true sphere, the UAV can land on inclined surfaces with relatively small inclinations. However, the UAV risks rolling over on surfaces with larger inclinations.

Luo et al. (2016) proposed a landing gear system for a UAV to accommodate inclined landings. The UAV itself does not incline and remains level, with the legs on the landing gear adjusting to the inclination.

In von Frankenberg and Nokleby $(2016,2017)$ the authors of this work presented a novel omni-directional quadrotor, the OmniCopter, that features a set of four rotors orthogonal to the four main lift rotors. Preliminary flight test results were presented in von Frankenberg and Nokleby (2016) and the OmniCopter's ability to reject disturbances was presented in von Frankenberg and Nokleby (2017). In neither of those papers was the ability of the OmniCopter to land on inclined surfaces was tested.

This paper extends the work of von Frankenberg and Nokleby $(2016,2017)$ to determine the OmniCopter's ability to land in a controlled manner on inclined surfaces. The ability to land on inclined surfaces is critical for the development of the AMS, since structures such as high-voltage power transmission towers often feature angled members. It should be noted that the ability to land in a controlled manner on an inclined surface is simply impossible in a traditional multirotor layout. For a traditional multirotor layout, the UAV must make a dynamic swooping-motion in order to land on an inclined surface. Such a dynamic swooping-motion landing would be extremely hazardous when attempting to land on a high-voltage transmission tower.

The outline of the remainder of the paper is as follows. Section 2 presents an overview of the OmniCopter. Section 3 discusses the control implementation used in this work. Section 4 presents the test results and a discussion of the results. The paper finishes with conclusions and recommendations for future work.

\section{OmniCopter}

For the OmniCopter, several rotor layouts were considered. Fig. 2a shows a top view of a relatively straightforward approach whereby four additional rotors with thrust axes directed orthogonal to the main-lifting rotors are added. The additional rotors in Layout 1 are in-line with the main lift rotors and are outwards of the main body of the quadrotor. Their axes of thrust pass through, or very close to, the centre of gravity of 
the UAV. Fig. 2b shows a similar approach except that the additional orthogonal-thrust rotors are contained internally in the space between the main lift rotors and are not in-line with the main cross-members of the frame. Their axes of thrust also pass through the centre of gravity of the UAV. Fig. 2c shows multi-view drawings of a rotor layout which contains 12 rotors and is essentially composed of three quadrotors bisecting each other in the $X-Y, Z-X$, and $Z-Y$ planes. Fig. 2 d shows a layout with four thrust rotors arranged in diagonally offset pairs. Fig. 2e shows a layout with only six rotors arranged in co-axial pairs on the $X, Y$, and $Z$ axes.

Layouts 3-5 were not selected due to their excessive mechanical complexity and the limited space in which accessories such as manipulators, docking mechanisms, and data-collection sensors could be mounted. It was predicted that Layout 1 would experience detrimental aerodynamic interactions between the additional thrust rotors and the main lift rotors. If the thrust rotors were activated they would move air directly above and beneath the main lift rotors, thus reducing their effectiveness and changing the amount of lift created depending on whether the thrust rotors are in use. This creates an additional control challenge. In comparison to Layout 2, Layout 1 is less space-efficient limiting how closely it can approach structures. Layout 2 was ultimately selected for further development, and is hereafter referred to as the OmniCopter.

\subsection{Equations of Motion}

The following section, which details the equations of motion of the OmniCopter, was developed by augmenting the equations of motion of a quadrotor, developed in Gibiansky (2012), to include the additional rotors. By defining the body-frame co-ordinate system and rotor numbering as shown in Fig. 3, the torque in the body frame $\left(\boldsymbol{\tau}_{B}\right)$ is determined as:

$$
\boldsymbol{\tau}_{B}=\mathbf{R}_{Z}\left(45^{\circ}\right)\left[\begin{array}{c}
L k\left(\omega_{1}^{2}-\omega_{3}^{2}\right) \\
L k\left(\omega_{2}^{2}-\omega_{4}^{2}\right) \\
b\left(\omega_{1}^{2}-\omega_{2}^{2}+\omega_{3}^{2}-\omega_{4}^{2}\right)
\end{array}\right]+\left[\begin{array}{c}
k_{t}\left(\omega_{7}\left|\omega_{7}\right|-\omega_{5}\left|\omega_{5}\right|\right) \\
k_{t}\left(\omega_{8}\left|\omega_{8}\right|-\omega_{6}\left|\omega_{6}\right|\right) \\
0
\end{array}\right]
$$

where $\mathbf{R}_{Z}$ rotates the original body-torque matrix used from Gibiansky (2012) about the $Z$ axis to align it with the coordinate system shown in Fig. $3, \omega_{i}$ is the angular velocity of the $i^{t h}$ rotor, $k$ and $k_{t}$ are constants of proportionality accounting for motor and propeller properties for the main rotors and orthogonal thrust rotors, respectively, $L$ is the distance from the centre of mass to the centre of one of the main lift rotors, and $b$ is a proportionality constant which accounts for the properties of the motors and propellers as they relate to torque.

The thrust force in the body-frame $\left(\mathbf{F}_{B}\right)$ is given as: 


$$
\mathbf{F}_{B}=\left[\begin{array}{c}
k_{f}\left(\omega_{5}\left|\omega_{5}\right|+\omega_{7}\left|\omega_{7}\right|\right) \\
k_{f}\left(\omega_{6}\left|\omega_{6}\right|+\omega_{8}\left|\omega_{8}\right|\right) \\
k \sum_{i=1}^{4} \omega_{i}^{2}
\end{array}\right]
$$

where $k_{f}$ is a coefficient that relates the propeller speed to thrust. The drag force $\left(\mathbf{F}_{D}\right)$ can be approximated to be proportional to the vehicle's air velocity and is given as:

$$
\mathbf{F}_{D}=\left[\begin{array}{c}
-k_{d x} \dot{x} \\
-k_{d y} \dot{y} \\
-k_{d z} \dot{z}
\end{array}\right]
$$

where $k_{d x}, k_{d y}$, and $k_{d z}$ are drag coefficients and $\dot{x}, \dot{y}$, and $\dot{z}$ are the translational velocities of the quadcopter in the $X, Y$, and $Z$ directions, respectively. The equations of motion for the centre of mass are therefore:

$$
m \ddot{\mathbf{x}}=\left[\begin{array}{c}
0 \\
0 \\
-m g
\end{array}\right]+\mathbf{R F}_{B}+\mathbf{F}_{D}
$$

where $m$ is the mass of the quadcopter, $\ddot{\mathbf{x}}$ is the acceleration of the quadrotor (with $\mathbf{x}=\left[\begin{array}{ll}x y & z\end{array}\right]^{\mathrm{T}}$ ), $g$ is the acceleration due to gravity, and $\mathbf{R}(\phi, \theta, \psi)$ is a rotation matrix relating the body frame to the inertial frame and is a function of the roll $(\phi)$, pitch $(\theta)$, and yaw $(\phi)$ angles.

\subsection{OmniCopter Prototype}

A physical prototype, shown in Fig. 4, was constructed and tests were conducted to determine the suitability of such a rotor layout as a base for an aerial mobile manipulator platform. The OmniCopter uses variable speed rotors, and can reverse the direction of rotor rotation to reverse the direction of thrust. A variable pitch rotor would use a mechanism to adjust the pitch of the rotor blades in order to control the magnitude of thrust, while keeping rotor speed at a maximum. Variable pitch rotors can change the magnitude of thrust more quickly than variable speed rotors, whereas variable speed rotors are mechanically simpler, cheaper, lighter, easier to maintain, and more reliable.

Each co-axial pair of thrust rotors is counter-rotating, and contains one clock-wise oriented propeller and one counter-clockwise oriented propeller. This was done so that the torque generated by the rotation of one rotor can be counteracted by the other rotor of that rotor pair. Since the propellers have opposite orientations, thrust is generated in the same direction while the rotors spin in opposite directions. A torque can be 
generated if the rotors spin in the same direction, while a net-zero thrust is generated. The sum of rotation rates determines net torque while the difference in rotation rates determines net thrust. Combinations of rotor speeds can be selected in order to generate desired combinations of torque and thrust.

Since the main lift rotors can be used to generate torques about all three axes independently, the addition of the thrust rotors gives the OmniCopter redundant control of torque for the roll and pitch axes. This means the OmniCopter could tolerate failure of some of the rotors.

A 2 meter long dual-conductor 14 AWG stranded copper cable was used to supply power from the battery to the prototype. The battery rested on the ground near the flight operating area.

Specifications for the prototype are presented in Table 1.

\section{Control Implementation}

An overview of the Control Implementation is shown in Fig. 5. A modified motion capture system, called the BlackTrax Tracking System, was used to measure the position of several infrared (IR) LEDs on the UAV. The software element of the BlackTrax system runs on a PC called the Tracking PC. The measured LED positions are fed back to another PC, called the Control PC, over wired Ethernet LAN. The Control PC calculates the control signals, which are then sent via USB to the Serial Pulse Position Modulation (PPM) Converter. The Serial PPM Converter converts the control signals from USB to PPM. The control signals are then fed into the RC (Radio Control) Transmitter Module. The RC Transmitter Module transmits the control signals over a $2.4 \mathrm{GHz}$ wireless radio protocol. The RC Transmitter Module also accepts manual control inputs from a human overseer. The human overseer can allow the control signals from the Control PC to pass through the RC Transmitter Module by activating a foot switch or take manual control of the UAV through the RC Transmitter Module's control joysticks. When the foot switch is released, the UAV is under manual control. The UAV receives the wireless control signals from the RC Transmitter Module and controls the velocity of its rotors. The UAV rotor speeds influence its position and orientation. The Control PC is informed whether manual control is activated by the RC Transmitter Module. This takes place over the same USB link as the control signals transfer over. It is shown using a separate arrow in the diagram for clarity.

This system was used to measure the position of two points on the body of the UAV in order to determine its position, yaw-angle, and pitch-angle (used only for plotting the pitch of the OmniCopter). The roll-angle could not be measured by tracking only two points. While the yaw-angle obtained by tracking these two points was used in the outer control loop, the roll and pitch angles were measured by an on-board IMU and controlled by the on-board flight controller. Fig. 3 depicts the position of the tracked points on the 
prototype. The tracking system consists of an Optitrack motion capture system, produced by NaturalPoint, augmented with BlackTrax hardware produced by CAST Group (see Fig. 6). The BlackTrax hardware augmentation improves the performance of the motion capture system. BlackTrax hardware consists of beacons and a timekeeper. One BlackTrax beacon can support three infrared IR LEDs. The BlackTrax timekeeper ensures that the beacons' LEDs pulse in sync with the frame rate of the Optitrack cameras by transmitting a synchronization signal to the beacons. Each camera's shutter opens in sync with the other cameras and during the time that the cameras' shutters are open, the IR LEDs of the beacons either turn on or stay off. The IR LED's state during each frame represents one bit of a binary code. Across several consecutive frames of camera data, each LED is able to transmit a unique code which allows the LED to be distinguished from other LEDs.

The Optitrack software application which calculates the position of tracked points based on camera data is called Motive. Motive transmits the positions of the tracked points via a proprietary IP protocol called NatNet. An software development kit to facilitate use of the protocol is made available by NaturalPoint.

The control of the OmniCopter is established as follows: 1) A desired velocity is calculated based on the difference between the OmniCopter's position and a goal position. 2) The desired velocity is proportional to the distance to the goal. 3) A maximum speed is also specified to avoid large velocities when the goal position is far away. 4) A nested PID control strategy is used to achieve these desired velocities. Further details on the control implementation can be found in von Frankenberg and Nokleby (2016).

\section{Test Results and Discussion}

As mentioned previously, preliminary testing of the OmniCopter was presented in von Frankenberg and Nokleby $(2016,2017)$. In this work, testing was done to determine the OmniCopter's ability to land in a controlled manner on inclined surfaces.

To test the OmniCopter's ability to land on inclined surfaces, two sets of tests were performed: the first with the landing surface at $20^{\circ}$ and the second with the landing surface at $30^{\circ}$. In these tests, the OmniCopter autonomously took off from rest, rose to a point $1.2 \mathrm{~m}$ above the takeoff position, then flew horizontally while maintaining a level orientation to a point above the inclined surface. Once above the inclined surface, the OmniCopter pitches to approximately $15^{\circ}$ using the thrust rotors to maintain position. While maintaining this pitch angle, the OmniCopter descended and landed on the inclined surface. Once it had landed, the OmniCopter then reversed the sequence of moves to return to the take-off point.

Fig. 7 presents an action-sequence image of the OmniCopter landing on the $20^{\circ}$ inclined surface. As can be seen in the series of images, the OmniCopter performs the required motion. The OmniCopter approaches 
the landing surface while maintaining a horizontal orientation. It then pitches to approximately $15^{\circ}$ and then proceeds to descend while maintaining the pitch angle, until it successfully lands on the $20^{\circ}$ inclined surface. This test demonstrates the increased flexibility of the OmniCopter as opposed to a traditional multirotor UAV. A traditional multirotor would risk the rotors striking the inclined surface during landing.

A second test was performed, this time with the inclined surface at an angle of $30^{\circ}$ to the ground. Fig. 8 presents an action-sequence of the test and Figures 9 and 10 present plots of the position, desired position, velocity, desired velocity, pitch angle, and control signals versus time. As can be seen in the figures, the OmniCopter sustains a pitch angle of $15^{\circ}$ as it descends. In this second test it was necessary to keep the orthogonal thrusters engaged once the OmniCopter had landed due to the greater angle of the incline. This allowed the OmniCopter to maintain stability on the surface (preventing it from sliding down the surface) and controlled the pitching moments induced by contact of the landing skids with the surface. As before, such a landing in a controlled manner would simply be impossible for a traditional multirotor.

The test results verified the OmniCopter's ability to land on inclined surfaces in a controlled manner. This ability is critical for the development of UAVs to perform maintenance tasks on tall structures such as high-voltage transmission towers.

\section{Conclusions and Future Work}

As part of a larger project for aerial inspection, construction, and maintenance of industrial structures, a UAV was required which could support equipment and application as an aerial mobile manipulator base. UAVs in these applications experience unpredictable aerodynamic forces due to the proximity of structures and must be capable of resisting forces and torques which arise from use of the manipulator and physical contact with the environment. Since traditional multirotors and helicopters have limitations due to the coupling of orientation and translation, a novel UAV concept was required.

A multirotor based on a novel rotor layout was developed to satisfy the requirements of aerial mobile manipulation. This concept was dubbed the OmniCopter. The OmniCopter features the addition of four orthogonal thrust rotors to a traditional quadrotor layout. The addition of these rotors gives the OmniCopter the ability to generate forces in any axis independently of its orientation. It also features redundant control of torque along the $X$ and $Y$ axes, giving it the potential to tolerate the failure of some rotors. The OmniCopter prototype demonstrated the ability to sustain $15^{\circ}$ roll or pitch angles and autonomously fly to and land on a surface inclined by up to $30^{\circ}$. In future, larger roll or pitch angles should be obtainable with more powerful thrust motors. The ability to fly at non-standard orientations means that the OmniCopter is more capable of landing on or docking with inclined surfaces than traditional multirotors, which are limited by the need 
to maintain a tilt proportional to their velocity.

The next step in the overall project will be to equip the OmniCopter with a Universal Docking Device (UDD) to allow it to securely land and clamp on to structures such as high-voltage transmission towers that are typically made out of angle iron members. These members are often at various angles, so the OmniCopter's ability to land on inclined surfaces will be critical to safely docking to such structures.

\section{Acknowledgements}

The authors would like to thank the Natural Sciences and Engineering Research Council (NSERC) of Canada for providing financial support for this research.

\section{References}

Albers, A., Trautmann, S., Howard, T., Nguyen, T. A., Frietsch, M., and Sauter, C. (2010). SemiAutonomous Flying Robot for Physical Interaction with Environment. In Proceedings of the 2010 IEEE Conference on Robotics, Automation and Mechatronics, pages 441-446.

Baltovski, T., Nokleby, S. B., and Pop-Iliev, R. (2013). Design and Development of an Swinging Arm Mechanism for an Aerial Manipulator System. In Proceedings of the International Conference on Mechanical Engineering and Mechatronics, Toronto, Canada.

Baltovski, T., Nokleby, S. B., and Pop-Iliev, R. (2015). Towards Performing Remote Manipulation Using an Autonomous Aerial Vehicle. In Proceedings of the 2015 CCToMM Symposium on Mechanisms, Machines, and Mechatronics, Ottawa, Canada.

Brescianini, D. and D'Andrea, R. (2016). Design, Modeling and Control of Omni-Directional Aerial Robot. In Proceedings of the 2016 IEEE International Conference on Robotics and Automation.

Cano, R., Pérez, C., Pruano, F., and Ollero, A. (2013). Mechanical Design of a 6-DOF Aerial Manipulator for Assembling Bar Structures Using UAVs. In Proceedings of the 2nd IFAC Workshop on Research, Education, and Development of Unmanned Aerial Systems.

Das, P. I. T. M., Swami, S., and Conrad, J. M. (2012). An Algorithm for Landing a Quadrotor Unmanned Aerial Vehicle on an Oscillating Surface. In Proceedings of the 2012 IEEE Southeastcon, pages 1-4.

Dougherty, J., Lee, D., and Lee, T. (2014). Laser-Based Guidance of a Quadrotor UAV for Precise Landing on an Inclined Surface. In Proceedings of the 2014 American Control Conference, pages 1210-1215. 
Gibiansky, A. (2012). Quadcopter Dynamics, Simulation, and Control Introduction Quadcopter Dynamics.

Jiang, G. (2013). Dextrous Hexrotor UAV Platform. Master's thesis, University of Denver.

Langkamp, D., Roberts, G., Scillitoe, A., Lunnon, I., Llopis-Pascual, A., Zamecnik, J., Proctor, S., Rodriguez-Frias, M., Turner, M., Lanzon, A., and Crowther, W. (2011). An Engineering Development of a Novel Hexrotor Vehicle for 3D Applications. In Proceedings of the International Micro Air Vehicles Conference.

Long, Y. and Cappelleri, D. J. (2013). Omnicopter: A Novel Overactuated Micro Aerial Vehicle. In Advances in Mechanisms, Robotics and Design Education and Research, pages 215-216. Springer.

Luo, C., Li, X., Li, Y., and Dai, Q. (2016). Biomimetic Design for Unmanned Aerial Vehicle Safe Landing in Hazardous Terrain. IEEE/ASME Transactions on Mechatronics, 21(1):531-541.

Mizutani, S., Okada, Y., Salaan, C. J., Ishii, T., Ohno, K., and Tadokoro, S. (2015). Proposal and Experimental Validation of a Design Strategy for a UAV with a Passive Rotating Spherical Shell. In Proceedings of the 2015 IEEE/RSJ International Conference on Intelligent Robots and Systems (IROS), pages 12711278 .

Papa, U. and Core, G. D. (2015). Design of Sonar Sensor Model for Safe Landing of an UAV. In Proceedings of the 2015 IEEE Metrology for Aerospace (MetroAeroSpace), pages 346-350.

Salazar, S., Romero, H., Lozano, R., and Castillo, P. (2009). Modeling and Real-Time Stabilization of an Aircraft Having Eight Rotors. Journal of Intelligent and Robotic Systems, 54(1-3):455-470.

Vlantis, P., Marantos, P., Bechlioulis, C. P., and Kyriakopoulos, K. J. (2015). Quadrotor Landing on an Inclined Platform of a Moving Ground Vehicle. In Proceedings of the 2015 IEEE International Conference on Robotics and Automation (ICRA), pages 2202-2207.

von Frankenberg, F. and Nokleby, S. B. (2016). Development of the OmniCopter: A Highly Maneuverable Quad-Copter Platform for the Aerial Manipulator System. In Proceedings of the Fourth Joint International Conference on Multibody System Dynamics, Montréal, Canada.

von Frankenberg, F. and Nokleby, S. B. (2017). Disturbance Rejection in Multi-Rotor Unmanned Aerial Vehicles Using a Novel Rotor Geometry. In Proceedings of the 4th International Conference of Control, Dynamic Systems, and Robotics, Toronto, Canada. 
Table 1: Specifications for the OmniCopter prototype.

\begin{tabular}{|l|l|}
\hline \multicolumn{2}{|c|}{ Prototype Specifications } \\
\hline \hline Mass & $1,513 \mathrm{~g}$ \\
\hline Frame diameter & $620 \mathrm{~mm}$ \\
\hline Main rotor diameter & $279.4 \mathrm{~mm}$ (11 inches) \\
\hline Main rotor pitch & $114.3 \mathrm{~mm}$ (4.5 inches) \\
\hline Maximum lifting thrust & $3.7 \mathrm{kgF}$ \\
\hline Thrust rotor diameter & $152.4 \mathrm{~mm}$ (6 inches) \\
\hline Thrust rotor pitch & $114.3 \mathrm{~mm}$ (4.5 inches) \\
\hline Maximum horizontal thrust & $1.0 \mathrm{kgF}$ \\
\hline Power source & Lithium-Polymer battery \\
\hline Battery voltage & $14.8 \mathrm{~V}$ \\
\hline Battery capacity & $10,000 \mathrm{mAh}$ \\
\hline
\end{tabular}




\section{Figure Captions}

Figure 1: Overview of the AMS project Baltovski et al. (2015).

Figure 2: Rotor layouts.

Figure 3: Definition of OmniCopter body-frame and rotor numbering along with the location of the two tracking markers (red circles).

Figure 4: OmniCopter prototype.

Figure 5: Overview of control implementation.

Figure 6: Motion capture testing area Baltovski et al. (2015).

Figure 7: An action-sequence of the OmniCopter landing on an inclined surface (20 incline).

Figure 8: An action-sequence of the OmniCopter landing on an inclined surface (30 incline).

Figure 9: Plots of position, pitch angle, and control signals for autonomous landing on an inclined surface (30 incline).

Figure 10: Plots of velocity, pitch angle, and control signals for autonomous landing on an inclined surface (30 incline). 


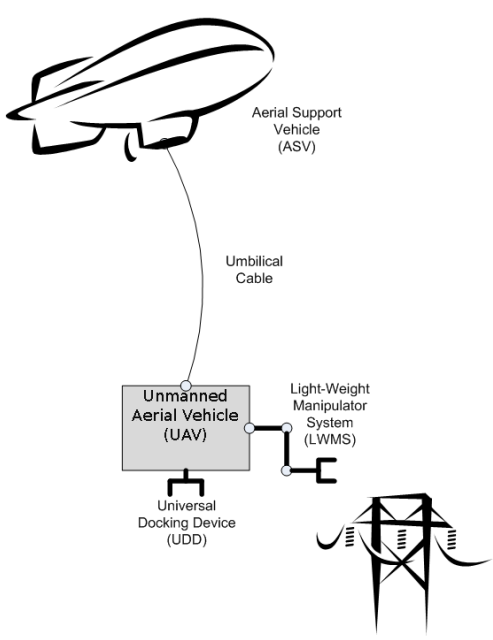

Figure 1: Overview of the AMS project Baltovski et al. (2015). 


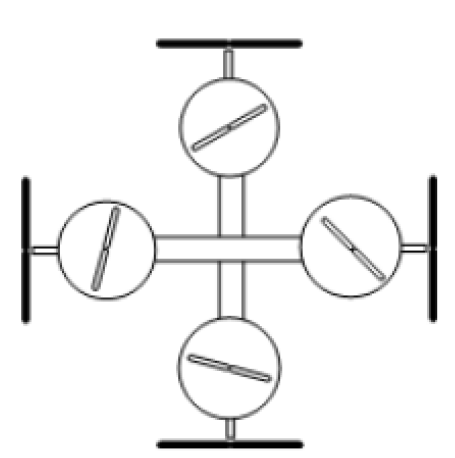

(a) Layout 1

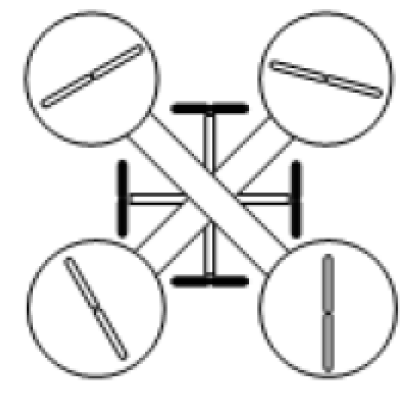

(b) Layout 2

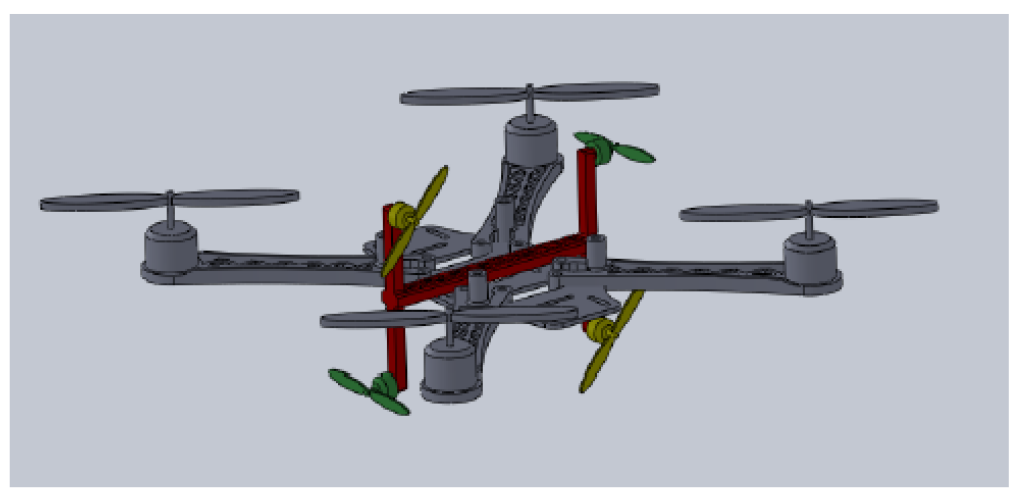

(d) Layout 4

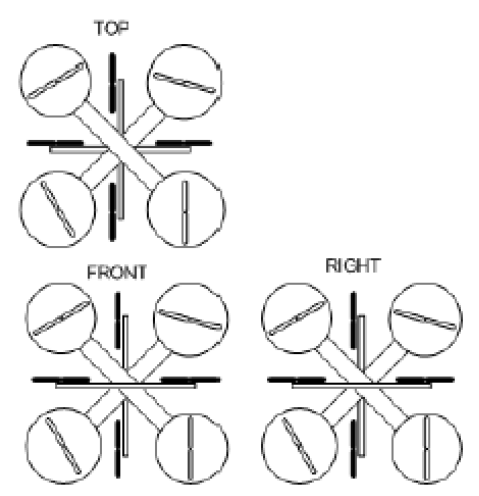

(c) Layout 3

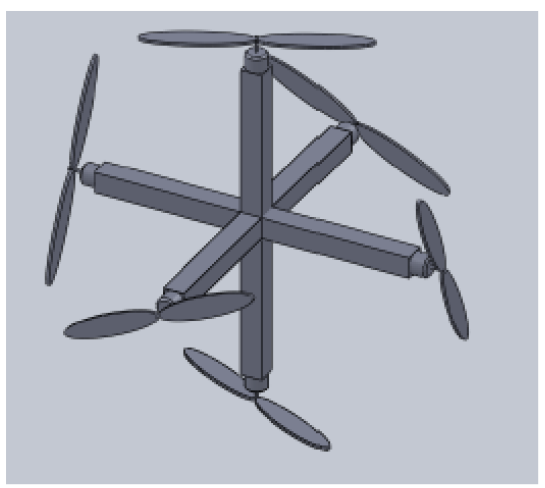

(e) Layout 5

Figure 2: Rotor layouts. 


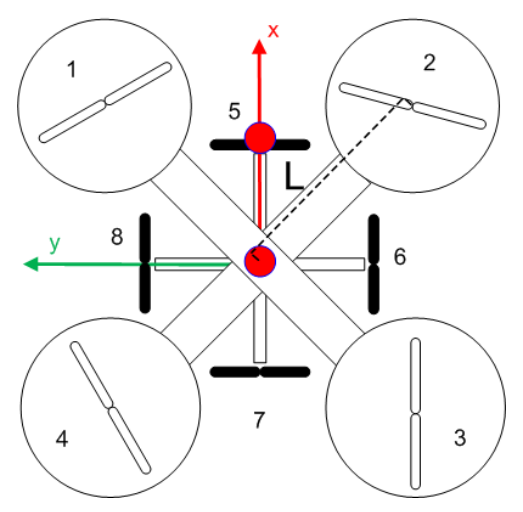

Figure 3: Definition of OmniCopter body-frame and rotor numbering along with the location of the two tracking markers (red circles). 


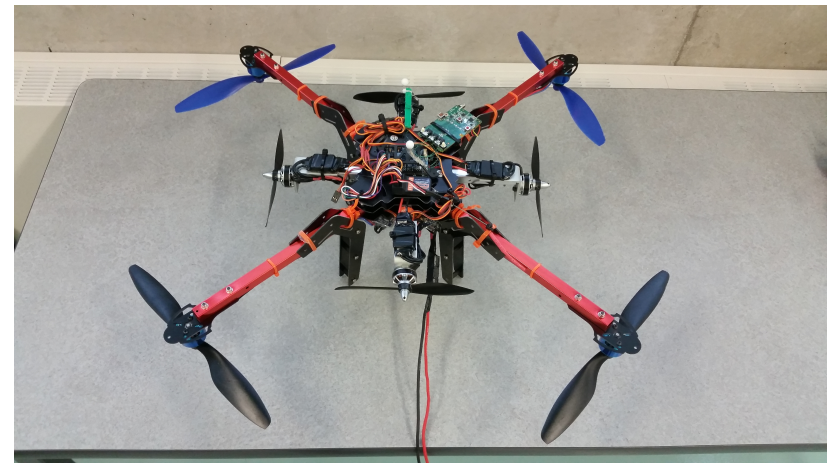

Figure 4: OmniCopter prototype. 


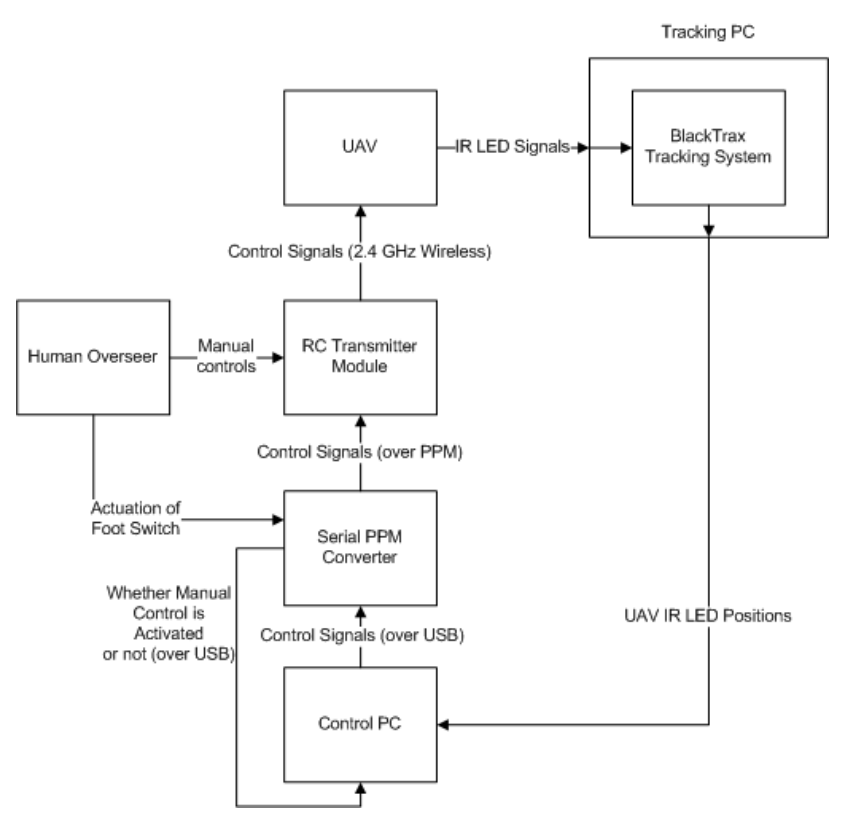

Figure 5: Overview of control implementation. 


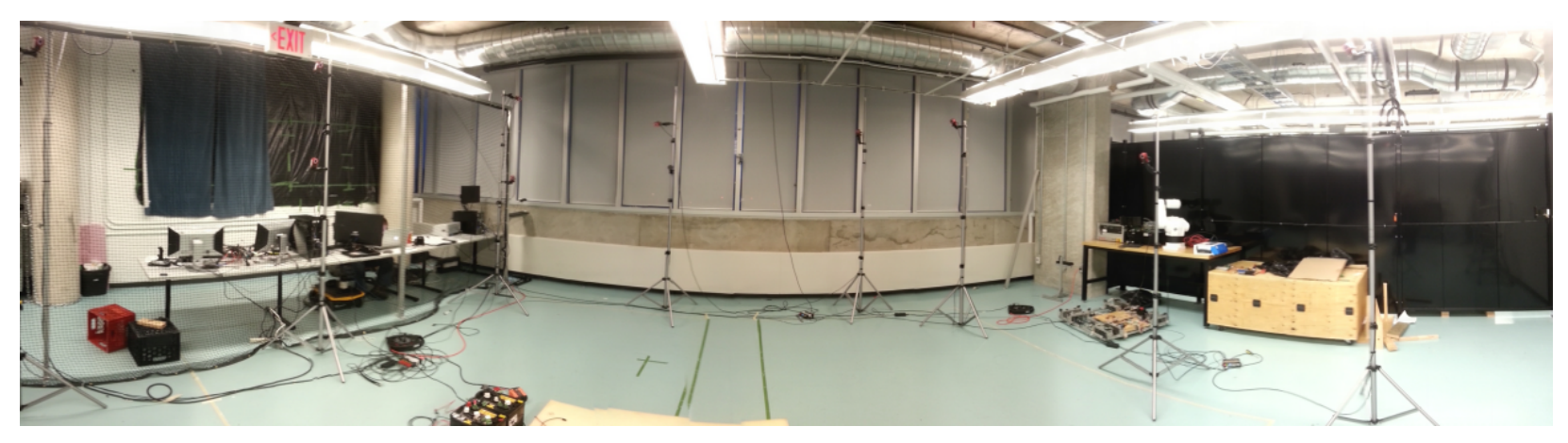

Figure 6: Motion capture testing area Baltovski et al. (2015). 


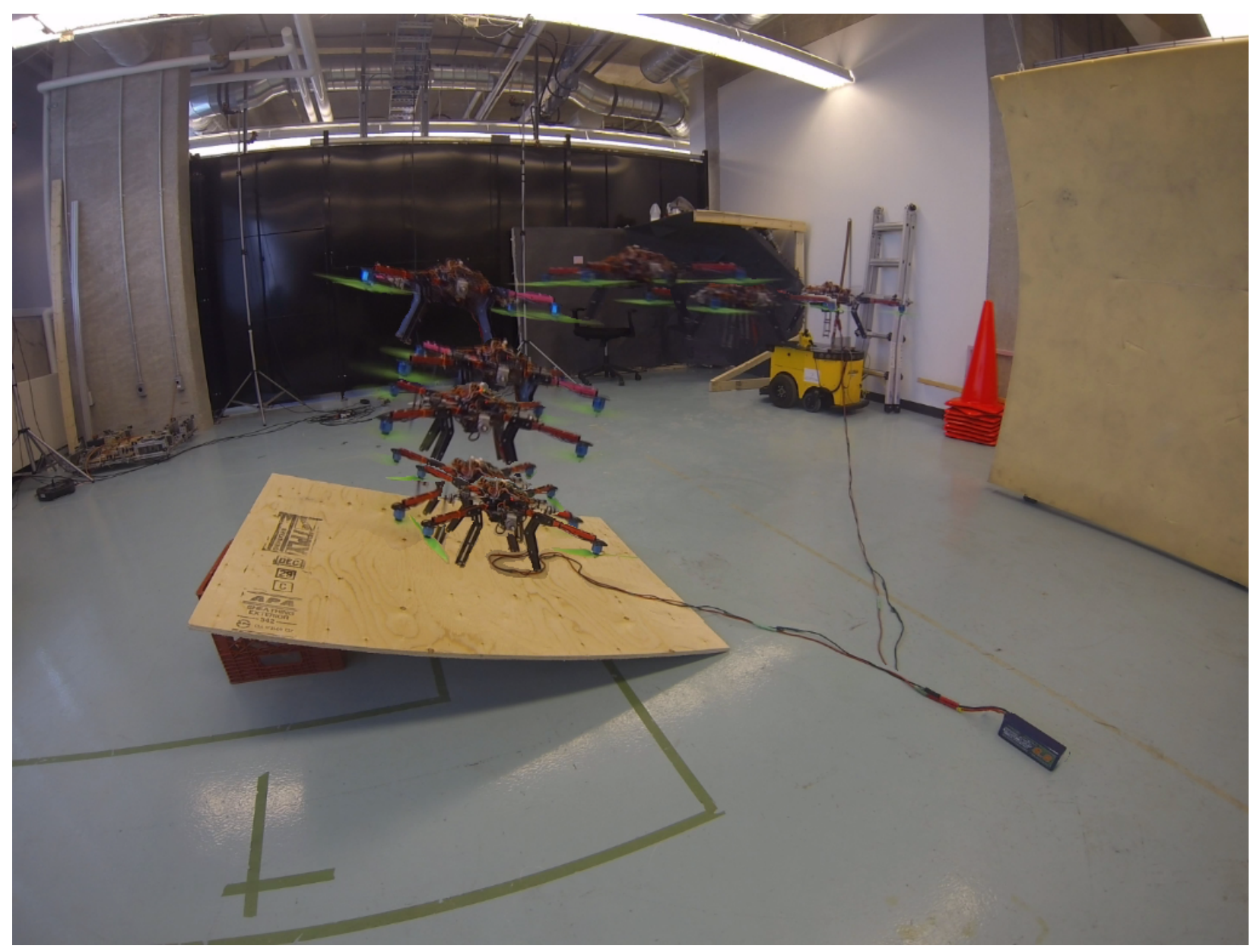

Figure 7: An action-sequence of the OmniCopter landing on an inclined surface (20 incline). 


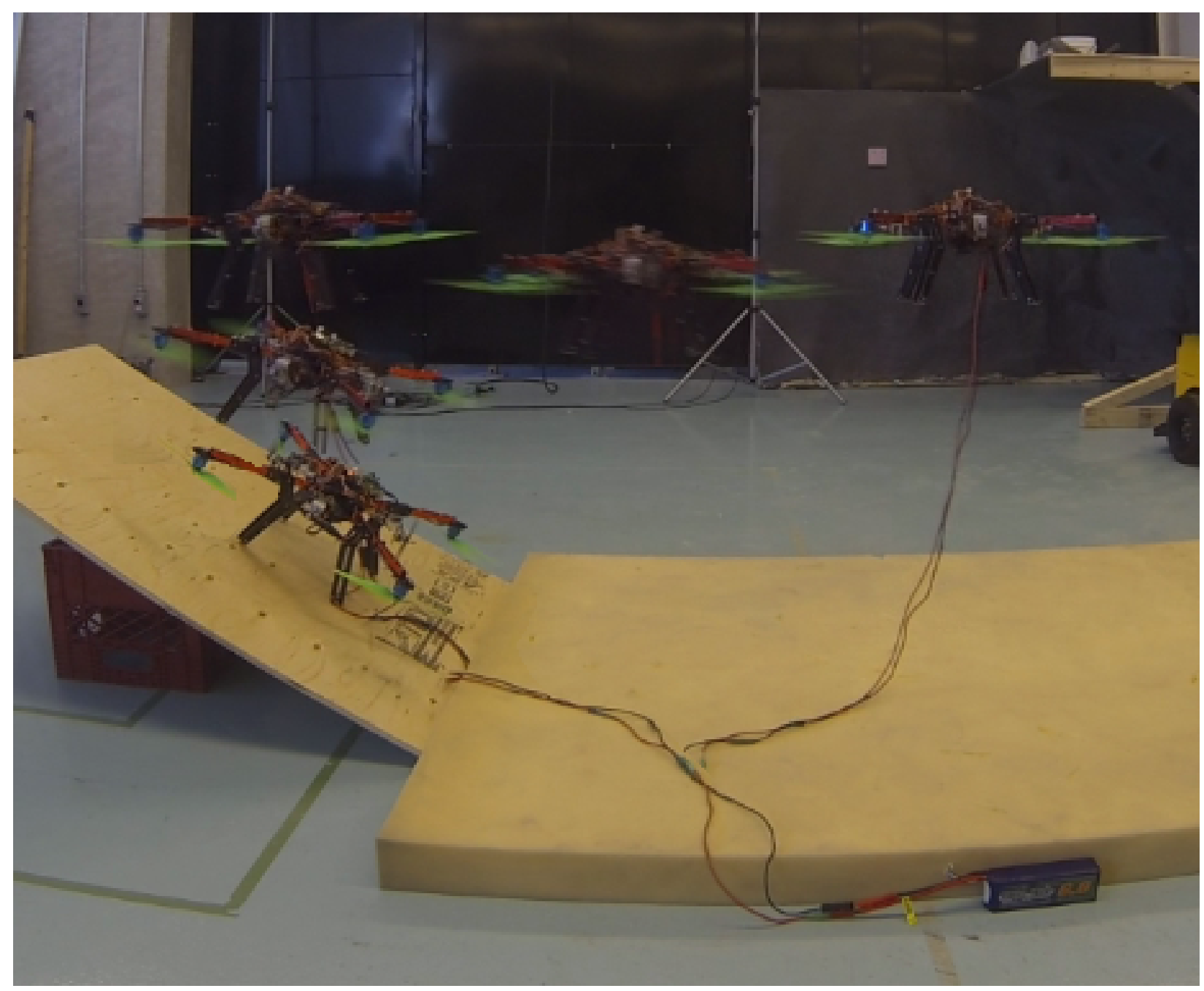

Figure 8: An action-sequence of the OmniCopter landing on an inclined surface ( $30^{\circ}$ incline). 


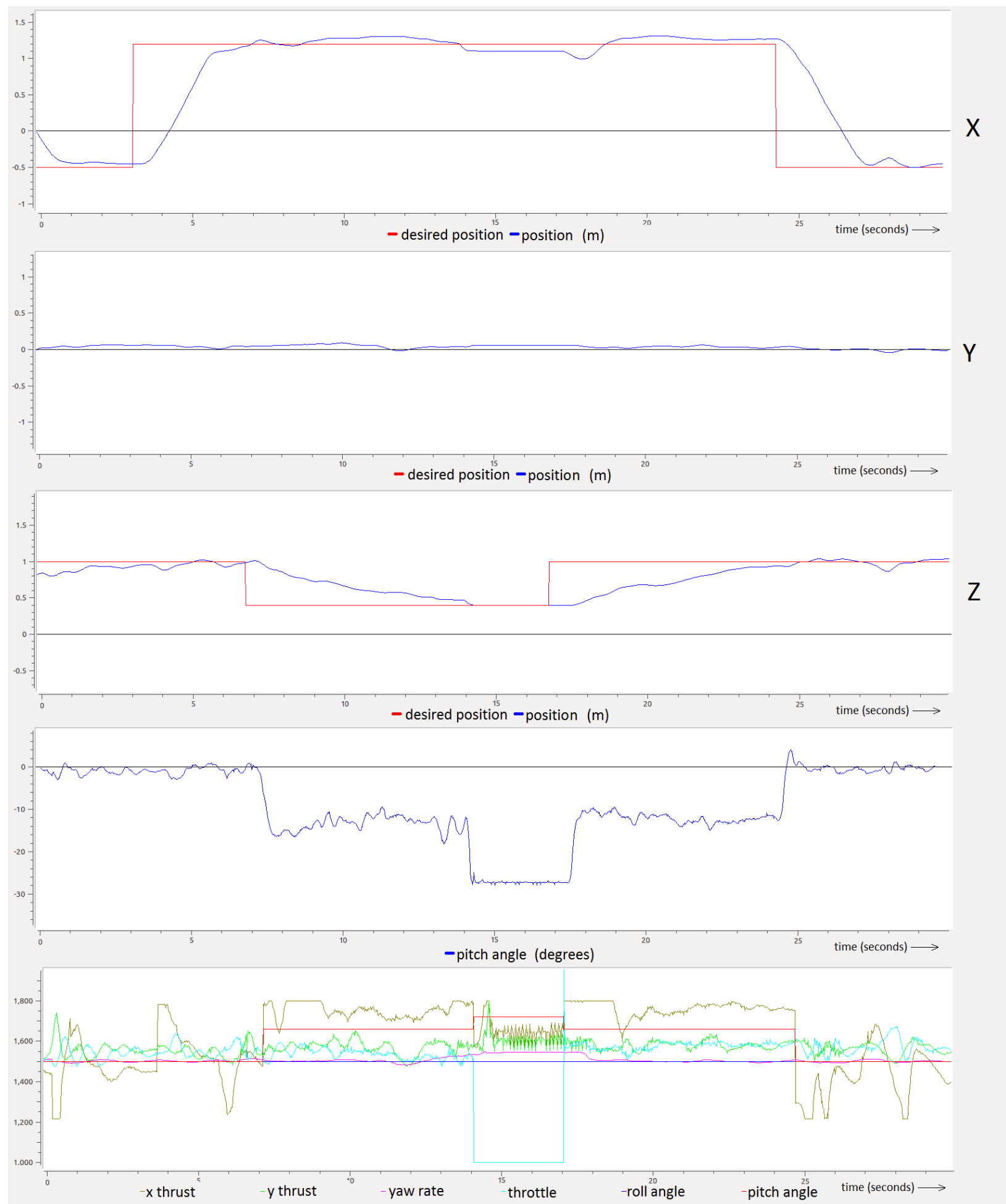

Figure 9: Plots of position, pitch angle, and control signals for autonomous landing on an inclined surface ( $30^{\circ}$ incline). 

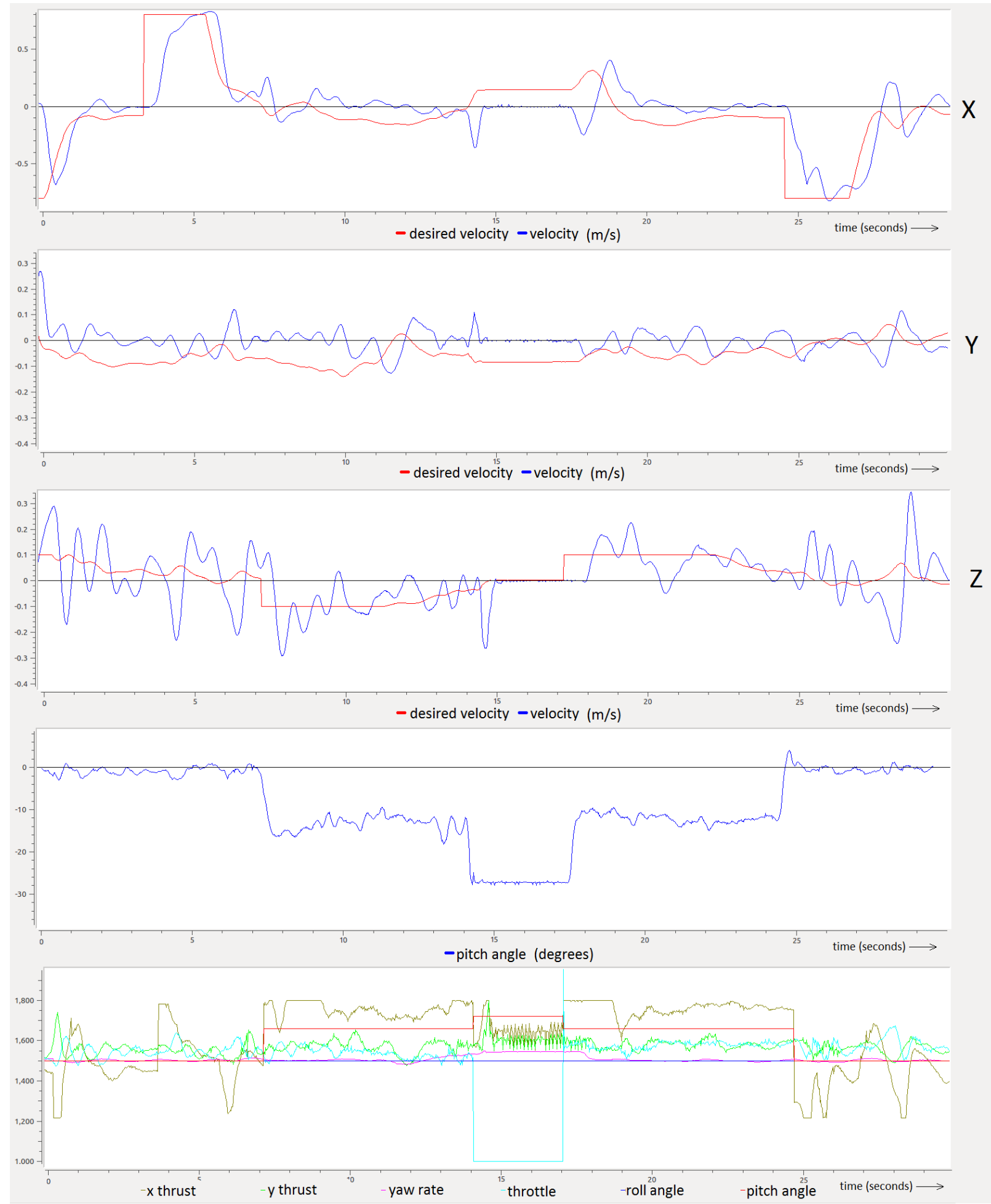

Figure 10: Plots of velocity, pitch angle, and control signals for autonomous landing on an inclined surface (30 incline). 